Résumés des conférences et travaux

\title{
L'édition des textes astronomiques grecs et byzantins
}

Anne Tihon

\section{(2) OpenEdition}

\section{Journals}

Édition électronique

URL : https://journals.openedition.org/ashp/657

DOI : 10.4000/ashp.657

ISSN : 1969-6310

Éditeur

Publications de l'École Pratique des Hautes Études

\section{Édition imprimée}

Date de publication : 1 octobre 2009

Pagination : 89-91

ISSN : 0766-0677

\section{Référence électronique}

Anne Tihon, «L'édition des textes astronomiques grecs et byzantins », Annuaire de l'École pratique des hautes études (EPHE), Section des sciences historiques et philologiques [En ligne], 140 | 2009, mis en ligne le 23 octobre 2009, consulté le 06 juillet 2021. URL : http://journals.openedition.org/ashp/657 ; DOI : https://doi.org/10.4000/ashp.657 


\title{
L'ÉDITION DES TEXTES ASTRONOMIQUES GRECS ET BYZANTINS
}

\author{
Conférences de $\mathrm{M}^{\mathrm{me}}$ Anne TiHon, \\ professeur à l'université catholique de Louvain, \\ Louvain-la-Neuve (Belgique), \\ directeur d'études invité,
}

\section{Première conférence}

Cette leçon avait pour thème les différents états dans lesquels un texte scientifique peut apparaître dans les manuscrits. À l'aide d'exemples, nous avons montré qu'un commentaire scientifique peut se rencontrer sous les formes suivantes :

a) Les notes prises au cours d'un enseignement oral. Ce cas a été illustré par le commentaire marginal qui se trouve dans les marges du Petit Commentaire de Théon dans le Paris. gr. 2394. L'histoire de ce manuscrit, copié en 1733 dans la bibliothèque de Constantin Maurogordato, a été retracée et le contenu du commentaire marginal présenté aux auditeurs : il s'agit d'un cours d'astronomie donné à Alexandrie à la

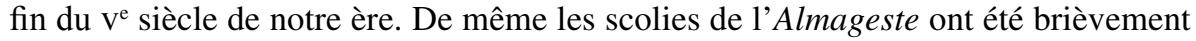
présentées.

b) Le deuxième stade est celui de la première rédaction, le brouillon non achevé. Ce cas a été illustré par le calcul d'éclipse du 16 juin 364 apr. J.-C. adapté de Théon d'Alexandrie et manifestement destiné à être intégré dans le Petit Commentaire de Théon. Mais ce projet n'a pas abouti car la majorité des manuscrits de ce traité ne contient pas ce calcul. Un deuxième cas a été présenté : celui de l'Introduction à l'Almageste, texte inédit, attribué par Joseph Mogenet à Eutocius d'Ascalon (fin $\mathrm{v}^{\mathrm{e}} \mathrm{s}$.).

c) L'édition faite par l'auteur : ce cas a été illustré par les Commentaires de Théon. On a défini les critères qui permettent de voir si un texte était bien destiné à la publication par son auteur : présence d'une préface avec dédicataire, cohérence interne, calculs sans fautes, démonstrations achevées, etc.

d) Les rééditions faites par l'auteur ou son entourage, qui se trahissent par la présence de renvois à des traités postérieurs.

e) Les éditions scolaires ou éditions alexandrines, illustrées par les mentions d'ecdosis dans les traités de Théon, d'Eutocius et d'Archimède; le rôle d'Hypatie, fille de Théon, dans l'édition des commentaires de son père.

f) Les recensions byzantines, plus faciles à déceler et illustrées par de nombreux exemples (Nicéphore Grégoras, Nicolas Cabasilas ...).

\section{Deuxième conférence}

Cette deuxième leçon a présenté de manière plus précise les traditions manuscrites et les problèmes d'édition des différents traités de Théon d'Alexandrie (ca 364 apr. J.-C.). 
1. Le Petit Commentaire de Théon. Ce traité, sorte d' «Astronomie pour les Nuls » de l'aveu même de l'auteur, explique le mode d'emploi des Tables faciles de Ptolémée en l'illustrant par des exemples. À cause de cela, ce traité a eu un grand succès et se présente dans cinquante-cinq manuscrits. Les différentes branches de la tradition ont été expliquées aux auditeurs, ainsi que le stemma codicum et les principes d'édition.

2. Le Grand Commentaire est un traité beaucoup plus difficile, attesté seulement dans douze manuscrits qui dérivent tous d'un exemplaire du $\mathrm{IX}^{\mathrm{e}}$ siècle, le Vaticanus gr. 190. Les scolies de ce traité dans ce manuscrit portent la date de 462 apr. J.-C. et mentionnent la ville d'Apamée où réside le scholiaste. Le texte y est dans un état extrêmement défectueux, ce qui a nécessité de nombreuses interventions d'éditeur.

3. Le Commentaire à l'Almageste, dont l'édition critique a été effectuée par A. Rome, mais seulement pour les livres I-IV. Le manuscrit le plus important suivi par l'éditeur est le Laurentianus 28/18, dont l'histoire a été présentée. À cette occasion, j'ai montré quelques documents (lettres du chanoine Rome) évoquant les difficultés à poursuivre les activités scientifiques à l'université de Louvain juste après la seconde guerre mondiale, alors qu'une partie de la bibliothèque universitaire avait été détruite et les notes relatives à Théon confisquées par la Gestapo, après l'arrestation de l'élève du chanoine Rome, l'abbé Joseph Mogenet.

L'édition du Commentaire à l'Almageste reste à terminer; le livre V, disparu, a été retrouvé dans les marges du Vaticanus gr. 198.

\section{Troisième conférence}

Cette leçon a été consacrée aux œuvres de Ptolémée. J'ai présenté l'auteur et ses œuvres, et ensuite les problèmes de la tradition manuscrite des Tables faciles, édition à laquelle je travaille avec Raymond Mercier (mathématicien et historien de l'astronomie, Cambridge). Il s'agit d'une œuvre astronomique qui comporte un mode d'emploi, un manuel rédigé par Ptolémée, et ensuite une série de tables astronomiques. Or, dans les manuscrits, le manuel a suivi la tradition de la Syntaxe mathématique (ou Almageste) qui est l'œuvre principale de Ptolémée, tandis que les tables ont suivi un chemin différent. L'édition des tables pose des problèmes particuliers, et j'ai présenté les solutions que nous avons adoptées. Les problèmes posés par les Hypothèses des planètes ont été brièvement esquissés. Ensuite les scolies de l'Almageste, particulièrement abondantes, inédites, ont été présentées : les scolies anciennes (scolies A) et les scolies récentes (scolies $\mathrm{B}$ ), avec les problèmes d'origine, d'identification des scoliastes, etc.

\section{Quatrième conférence}

Cette leçon avait pour objet l'édition des textes astronomiques byzantins. J'ai d'abord présenté le projet de Corpus des astronomes byzantins et son état d'avancement. La matière à éditer couvre approximativement soixante-six traités ou textes importants d'astronomie mathématique; dix-sept traités sur l'astrolabe; environ dix traités inclassables et de nombreux textes non encore inventoriés. Le $C A B$ compte dix volumes parus, mais on manque de collaborateurs compétents et surtout de ressources 
financières qui permettraient de continuer les éditions. J'ai présenté un projet de répertoire des textes, avec un essai de classification des documents astronomiques :

1. les traités rédigés; 2 . les tables (parfois associées au $n^{0} 1$ ); 3 . les éphémérides (rares dans le monde byzantin); 4. Les calculs isolés; 5. les scolies et notes isolées; 6. les figures et diagrammes. Cette dernière catégorie doit elle aussi être définie, car on doit distinguer : a. les figures géométriques; b. les figures astronomiques; c. les dessins d'instruments; d. les diagrammes; e. les thèmes astrologiques. Enfin quelques problèmes posés par des traités byzantins ont été présentés : le traité de Stéphane d'Alexandrie (thèse de doctorat en cours, par Jean Lempire), le traité sur la date de Pâques de Barlaam (en préparation), le manuel de Georges Gémiste Pléthon (édité dans le $C A B$ ). Des problèmes de méthodologie ont été discutés : la nécessité et l'importance d'identifier correctement les copistes (exemple dans le cas de Georges-Grégoire Chioniadès, Théodore Méliténiote), la méthode de collation et de classement des manuscrits (méthode de Joseph Mogenet). 